\section{Patent fights rumble in China}

\author{
Opinions differ in interpretations of patent prosecution and infringement litigation
}

Ken Howard

Two recent patent decisions in China against the top two pharma companies could be the murmurings of its patent system becoming more firmly entrenched in domestic economic growth, while international companies are attempting to achieve a favourable intellectual property position in a potentially huge market.

In August, GlaxoSmithKline voluntarily abandoned a formulation patent in China for its diabetes treatment rosiglitazone maleate (Avandia), after it was challenged by four Chinese companies. And Pfizer is appealing against a decision by China's patent review board, which in July revoked its method of use patent for Viagra.

"The [Viagra] patent was rejected because it didn't contain information that was not required or asked for" at the initial filing, says Bryant Haskins, a spokesperson at Pfizer. "We think this type of ruling undermines the patent process in China [and it] certainly gives us cause for concern."

China joined the World Trade Organization (WTO) three years ago, and since then its legal system has made enormous strides to harmonize its laws and procedures with other WTO countries.

The Chinese government's decisions on the Viagra and Avandia cases were based on Chinese patent law, says Rongling Deng, a World Health Organization fellow who is now with the Chinese regulatory authority, SDA, although her comments were not made in an official capacity.

"In 2001, Pfizer received the Viagra patent of method of use in a great argument," says Deng. "It was said there were political reasons, since from Chinese law's perspective, Pfizer should not have been granted this patent." Deng says this challenge is no different to when Pfizer lost the same Viagra patent in the UK to Lilly with Cialis.

"GSK gave up its formulation patent on rosiglitazone, but still holds the compound patent and the manufacturing process patent on Avandia until 2013," says Deng.

"I guess GSK made its decision because of the weak defences of novelty and fairness of the patent."

The recent cases could actually signal the emergence of a robust local patent system, says Ronald Eisenstein, a partner in the biotechnology and intellectual property group at Nixon Peabody, based in Boston, Massachusetts. "If you look at Taiwan, the patent system is notorious for decisions that give home court advantage to local companies," he says.

Because Chinese law is different than US, the Pfizer and GSK decisions might be correct based on the reading of local law, says Eisenstein. Additionally, he points out, if a patent is written on the basis of laws of the country from which it emerged, you can hit road blocks in other countries whose laws and processes differ even slightly.

From the Chinese market perspective, counterfeit drugs are still a problem that

\title{
Public molecules: small, but perfectly formed
}

New repositories aim to provide chemical biologists with the support to help answer molecular questions faster.

David Bradley

Two databases have been launched, which aim to bridge the gap between chemistry and biology using different approaches.

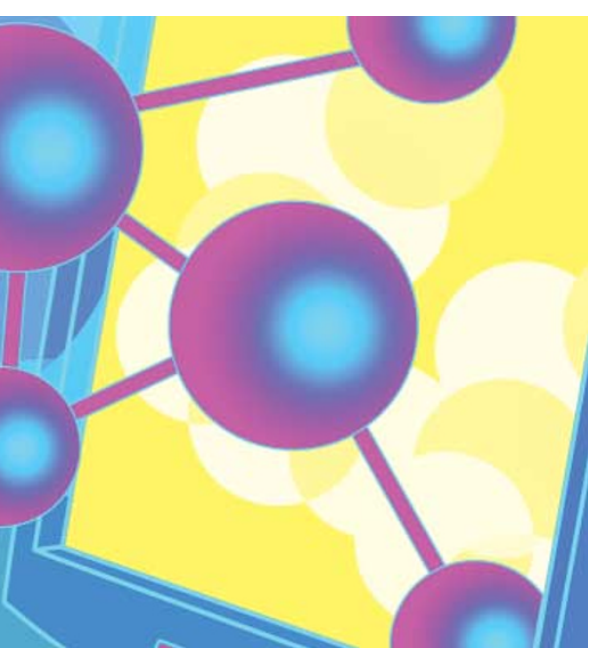

Two new chemical databases are freely available to researchers.
The first database, PubChem (http://pubchem.ncbi.nlm.nih.gov/), is part of the US National Institutes of Health's 'Molecular Libraries' initiative, which already has 650,000 diverse small molecules in its collection and a further 100,000 to be included in 2005. The collection will contain as many independent scaffolds as possible, natural products, all current FDA-approved drugs, and other compounds obtained from private and public sources. These will be sourced from NIH-funded small-molecule screening centres and elsewhere.

PubChem will provide biomedical researchers with access to structural, chemical and physical information, which they can screen computationally or experimentally. The information will allow researchers to pick a suitable small molecule as a probe for important cellular pathways involved in health and disease, as an imaging agent, or potentially as a drug lead.

Bioinformatician Neil Saunders of the University of New South Wales is impressed by how PubChem integrates into the National Center for Biotechnology Information's website/ database structure. "It will be of use primarily for bioassays," he says, "or in docking simulations." Its usefulness as a research tool will be in allowing researchers to systematically screen thousands of small molecules.

The second database, called Chemical Entities of Biological Interest (ChEBI; http:// www.ebi.ac.uk/chebi/) and produced by the European Bioinformatics Institute, contains naturally occurring and synthetic small molecular entities. However, the similarity to PubChem ends there. Whereas PubChem will provide a diverse database of potential drug leads and molecular probes, ChEBI is essentially a dictionary with a controlled vocabulary for looking up information about a chemical entity of interest.

"We see as our target audience those who use biological databases," explains curator Marcus Ennis. Moreover, the ontological aspect of ChEBI will allow EBI to record relationships between molecular entities or classes of entities in a defined way.

Each ChEBI entry contains details about the entity's chemistry and biological activity. Synonyms for each entity are also listed and are 
both international and local drug companies face, says Deng. "However, from the local drug maker perspective, international drug companies somehow have better treatment than local drug companies, especially international giants."

To help accelerate the harmonization process in China, the United States Patent and Trademark Office (USPTO) has for the first time stationed an attorney-advisor, Mark Cohen, overseas.

The USPTO's main goals with respect to pharmaceutical drugs are to work with the Chinese government to improve enforcement against counterfeit products; to help streamline China's patent application process; to assist in implementing laws and regulations to protect clinical data that must be submitted to regulatory agencies in China; and to help develop a system to ensure that government agencies in China are coordinating efforts against approval of generics on patented pharmaceutical products without the consent of the patent owner, says USPTO spokesperson Brigid Quinn.

All parties acknowledge that despite the difficulties China represents such a potentially huge market that this will motivate both local pharmaceutical companies, as well as international corporations, to continue to pay close attention to China's patent system.

searchable. The database includes the relationship between macromolecules, such as proteins, and small molecules via UniProt protein knowledgebase cross-links.

Although ChEBI is small, Ennis hopes that with improved funding the database will have scope to grow. "As the EBI databases begin to use ChEBI, its public awareness will increase, which hopefully will improve our prospects for increased funding."

Steve Bryant, Senior Investigator in charge of PubChem, said that they are more than open to data exchange and collaboration with related international efforts. "The projects are new, and I think it will take some time to sort out what we can best do together," says Bryant.

Issues of intellectual property regarding compounds should not be a problem, because a structure cannot be copyrighted even though its applications can be patented.

"Both databases appear to be good ideas attempting to address complex problems, but it is really too early to tell how useful they will be," says Daniel Weaver of Array BioPharma, a company based in Boulder, Colorado that specializes in informatics-led drug discovery. Curation of individual structures at each site will be key to their validity in a biological research environment.

Weaver says that the developers of both databases will soon face many of the complexity issues pharmaceutical companies have tried to address. Which compounds to include, how to handle chemical variations in stereochemistry and tautomers, and how to store and retrieve appropriate compound information for the user community, will all need to be addressed.

\section{NEWS IN BRIEF}

\section{More trouble for COX2 inhibitors}

Since the withdrawal of rofecoxib (Vioxx) in September, cyclooxygenase 2 (COX2) inhibitors have been placed under the microscope. Articles in the Wall Street Journal and Lancet accused Merck of knowing about the cardiovascular health risks associated with Vioxx years before pulling it from the market. On its website, Merck wrote a detailed critique of the Lancet article, claiming that early data on Vioxx showed no significant difference in cardiovascular risk between the drug and either a placebo or nonsteroidal anti-inflammatory drugs other than naproxen. Meanwhile, the US Justice Department has launched a criminal investigation into Merck's handling of Vioxx, and the US Securities \& Exchange Commission has begun an informal inquiry that is likely to focus on whether Merck provided investors with adequate information about Vioxx's risks. Pfizer's valdecoxib (Bextra) hasn't escaped the controversy either. The company said it's likely that a 'black box' warning will be added to Bextra's label because it can lead to a rare but serious skin reaction called Stevens-Johnson syndrome. Also, data presented at the American Heart Association meeting in New Orleans by Garret FitzGerald on patients with arthritis or who had had open-heart surgery suggested that the Bextra arm had more than double the number of heart attacks or strokes than the placebo arm. Pfizer released a statement saying these were unsubstantiated conclusions, as they improperly combined data from heart surgery patients and lower-risk arthritis sufferers.

\section{FDA to revaluate safety monitoring system}

In the wake of criticism over the US FDA's handling of warnings about Vioxx and antidepressants in children, the agency has announced a major review of its system for monitoring the safety of marketed drugs. In both cases, agency reviewers complained that their warnings about potential problems were ignored or suppressed before the risks became known publicly. The agency said it will sponsor an Institute of Medicine study of its safety-monitoring procedures, and will set up a formal programme to ensure that the views of all agency scientists are included in future procedures.

\section{GSK to move clinical trials to developing countries}

GlaxoSmithKline's CEO, Jean-Pierre Garnier, has outlined how the company is reducing its R\&D costs. GSK is moving up to $30 \%$ of its clinical trials to low-cost countries. Such a move can save GSK between US $\$ 10,000$ and $\$ 15,000$ per enrolled patient, and the $30 \%$ shift to low-cost countries could save more than $\$ 200$ million in annual R\&D costs. A fully operational electronic data-capture system would reduce the R\&D cost further, as this would decrease the number of data managers needed, said Garnier.

\section{Genasense loses support but keeps going}

Genta said that Aventis will terminate agreements to develop and commercialize its BCL2-antisense cancer drug oblimersen sodium (Genasense) on 8 May 2005. Earlier this year, an FDA advisory committee voted 13 to 3 that the progression-free survival data that Genta provided for oblimersen in breast cancer could not be considered substantial evidence of effectiveness. Genta said it intends to continue to develop oblimersen, and it revealed data from a study of 241 patients with relapsed or resistant chronic lymphocytic leukaemia. Oblimersen met the primary goal of showing a benefit compared with chemotherapy, but data so far do not show a significant difference in key secondary end-points, including time to progression and overall survival.

\section{The beginning of race-based medicine?}

Two clinical trials of cardiovascular drugs in black patients have raised the notion that we could be moving into a new era of race-based therapeutics. The African-American Heart Failure Trial (A-HeFT) showed a 43\% reduction in one-year mortality by adding NitroMed's BiDil nitric oxide therapy (isosorbide dinitrate/hydralazine) to the standard heart failure regimen for African-Americans (Taylor, A. L. et al. New Engl. J. Med. 351, 2049-2057 (2004)). The researchers propose that heart failure has a different pathophysiology in blacks than in whites, necessitating different treatment strategies. AstraZeneca also released the results of its African-American Rosuvastatin Investigation of Efficacy and Safety study (ARIES) in 774 black adults. This found that after six weeks of treatment, rosuvastatin (Crestor) reduced low-density-lipoprotein cholesterol levels by $46 \%$, whereas Lipitor (atorvastatin) at the same strength lowered levels by $39 \%$. news@nature.com www.nature.com/news
For more news and analysis go to 